aortic arch that surrounds the lung bud. It has been proposed that pulmonary artery sling arises when the left lung bud fails to connect with the left sixth arch and instead forms an anastomosis with the right sixth arch. If this occurs dorsal to the developing lung bud, it results in a left pulmonary artery arising from the right pulmonary artery, coursing posterior to the trachea, creating the sling. The same explanation may apply to the partial left pulmonary artery sling, in which the anomalous component courses behind the trachea because of a dorsal connection to the lung bud. If such a connection occurs ventral to the developing lung bud, this result in a partial anomalous left pulmonary artery. ${ }^{1}$

Anomalous left pulmonary artery is associated with other congenital heart defects in $40 \%$ to $50 \%$ of cases. Partial anomalous left pulmonary artery and partial left pulmonary artery sling have been described in association with ventricular septal defect, ${ }^{1}$ double-outlet right ventricle with unbalanced atrioventricular septal defect, ${ }^{2}$ coarctation of the aorta, ${ }^{2,3}$ and mitral stenosis. ${ }^{2,3}$ To our knowledge, associa- tion with "swiss cheese" ventricular septal defects has not previously been reported. Interestingly, Koch and colleagues $^{3}$ reported a case of partial anomalous left pulmonary artery in association with imperforate anus, as in our patient, suggesting an association with a more complex process of dysmorphogenesis and a genetic background. This is reinforced by the association of other congenital anomalies present in our patient. The fact that our patient did not have an obstructive airway is unusual in the setting of a partial left pulmonary artery sling, which encircles the lower trachea. The patient was discharged in good condition but will require careful follow-up of the pulmonary vasculature and central airways.

\section{References}

1. Erickson LC, Cocalis MW, George L. Partial anomalous left pulmonary artery: new evidence on the development of the pulmonary artery sling. Pediatr Cardiol. 1996; 17:319-21.

2. Fountain-Dommer RR, Shirali GS, Wiles HB, Larsen RL. Noninvasive diagnosis of partial anomalous left pulmonary artery. J Am Soc Echocardiogr. 2001;14:745-6.

3. Koch A, Hofbeck M, Gerling S, Buheitel G, Singer H. [Partial anomalous origin of the left pulmonary artery]. [German] Z Kardiol. 2000;89:118-21.

\title{
Cardiac tamponade secondary to traumatic intrapericardial diaphragmatic hernia
}

\author{
Affes Nejmeddine, MD, ${ }^{\mathrm{a}}$ Abdenader Mohamed, MD, ${ }^{\mathrm{b}}$ Khemakem Khalil, MD, ${ }^{\mathrm{c}}$ Ben Salah Khalil, MD, ${ }^{\mathrm{a}}$ and \\ Beyrouti Mohamed Issam, MD, ${ }^{a}$ Sfax, Tunisia
}

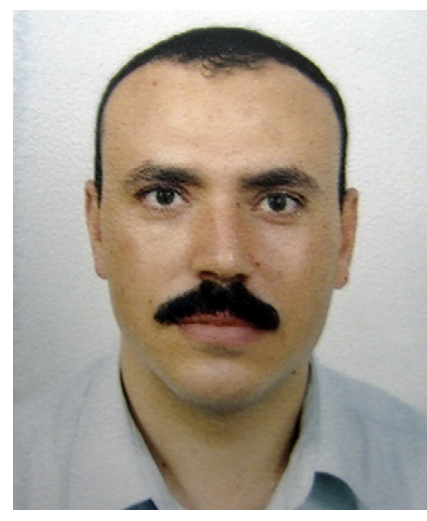

Dr Nejmeddine.

\footnotetext{
From the Departments of General Surgery, ${ }^{\mathrm{a}}$ Cardiac Surgery, ${ }^{\mathrm{b}}$ and Anesthesia, ${ }^{\mathrm{c}}$ Habib Bourguiba Hospital, Sfax, Tunisia.

Received for publication Oct 25, 2007; accepted for publication Dec 9, 2007.

Address for reprints: Affes Nejmeddine, MD (E-mail: affesnejm@yahoo.fr).

J Thorac Cardiovasc Surg 2008;136:1087-9

$0022-5223 / \$ 34.00$

Copyright (c) 2008 by The American Association for Thoracic Surgery

doi:10.1016/j.jtcvs.2007.12.075
}

An unusual case of cardiac tamponade secondary to traumatic intrapericardial diaphragmatic hernia is presented: a defect of the pericardium, with herniation of the stomach and the greater omentum into the pericardial sac. A preoperative diagnosis was suspected by clinical and chest x-ray signs and confirmed by computed tomography (CT). A laparotomy was quickly performed. Successful operative repair of the tear was performed with interrupted reabsorbable sutures. The case is discussed, and the management of patients with these rare lesions is reviewed.

Intrapericardial diaphragmatic hernia results from a posttraumatic and severe compressive injury of the chest and abdomen. Herniation into the pericardium is rare; approximately 1 posttraumatic intrapericardial hernia is reported every year. We review the posttraumatic intrapericardial diaphragmatic hernia cases in the literature to discuss the diagnostics and management of this lesion.

\section{CLINICAL SUMMARY}

A 56-year-old man was involved in a car crash that caused a bilateral costal fracture with blunt abdominal trauma. The 


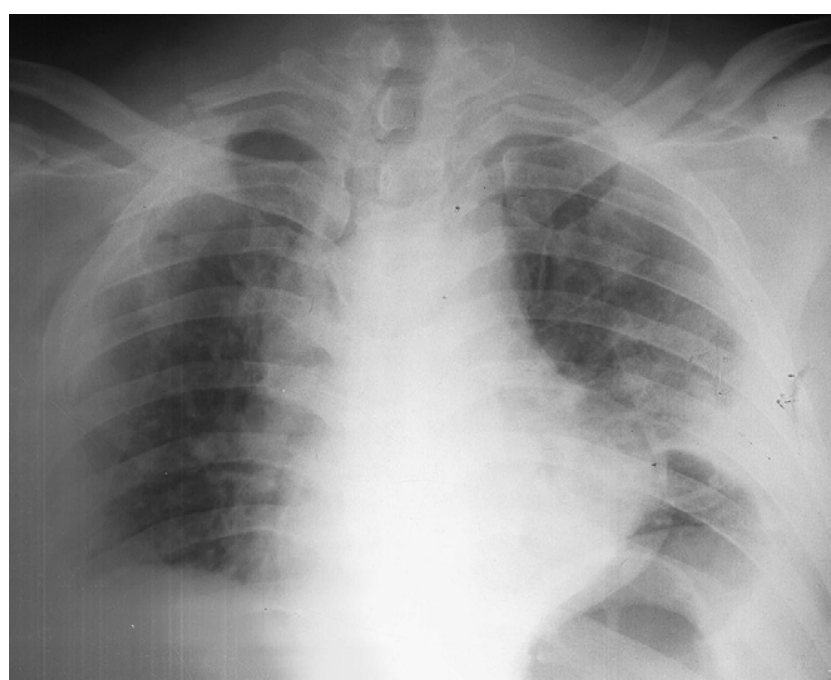

FIGURE 1. The left hemidiaphragm is not seen. There is a supradiaphragmatic air collection.

patient reported diffuse abdominal pain, and the abdomen was tender on examination.

The patient's vital signs were as follows: heart rate of 140 beats/min; arterial blood pressure of $110 / 65 \mathrm{~mm} \mathrm{Hg}$; and respiratory rate of 32 breaths/min. Initial laboratory testing revealed a hemoglobin level of $11 \mathrm{~g} / \mathrm{dL}$ and a white blood cell count of $11,000 / \mathrm{mm}^{3}$. A chest $\mathrm{X}$-ray showed multiple bilateral rib fractures. A supradiaphragmatic air collection was noted in the left side of the heart.

A diaphragmatic rupture with herniation into the pleural cavity was initially suspected (Figure 1). CT of the chest and abdomen demonstrated a contusion of the left lower lobe, mild bilateral hemothorax, and displacement of the heart to the right side. CT also showed a digestive organ on the left side of the heart, resembling intrapericardial stomach located with, (Figure 2) and a splenic and hepatic trauma.

During the next few hours, the patient had multiple episodes of hypotension and periods of tachycardia and bradycardia, with distended neck veins. The patient was believed to have an intrapericardial diaphragmatic hernia. Because the patient's hemodynamic status was deteriorating, he was referred for an immediate laparotomy. At surgery, an emergency midline laparotomy revealed a $10-\mathrm{cm}$ defect in the central portion of the diaphragm, in which the greater omentum and the stomach were herniated. The replacement of the hernia contents led to immediate hemodynamic improvement. The left pleural cavity was without defect. In addition, there was a retroperitoneal hematoma, hemoperitoneum, mesentery laceration, and hepatic and splenic benign contusion. Careful examination of the heart revealed no myocardial contusions. A drainage tube was placed in the pericardium. The central tendon was repaired with interrupted sutures. The patient's hemodynamic status was stable immediately after surgery.

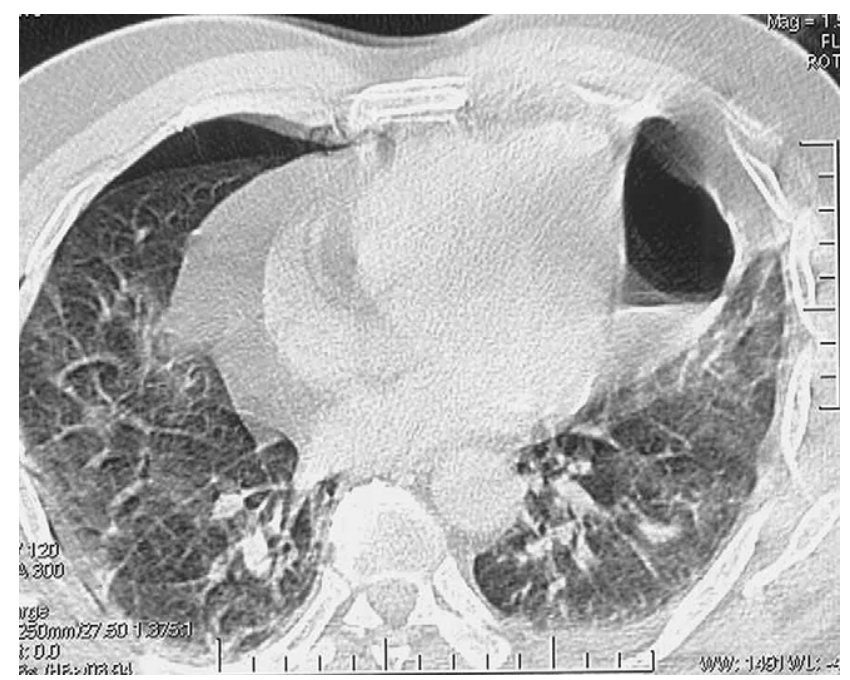

FIGURE 2. CT reveals a digestive structure in the pericardium.

\section{DISCUSSION}

Diaphragmatic ruptures are caused by a severe abdominal trauma during which the abdominal pressure increases to at least $1000 \mathrm{~cm} \mathrm{H}_{2} \mathrm{O} .{ }^{1}$ In 1910 , the first report of a traumatic intrapericardial diaphragmatic hernia in the autopsy findings of 2 cases. $^{2}$

Most traumatic intrapericardial diaphragmatic hernias are caused by blunt trauma of the chest, abdomen, or both. A car crash $(71.4 \%)$ is the most usual mode of injury. The diagnosis of the intrapericardial diaphragmatic hernia was immediate in $48 \%$ of cases or delayed with an interval time from 1 month to 23 years. ${ }^{3}$

The most frequent clinical symptoms are respiratory in $40 \%$ of cases and abdominal in $38 \%$ of cases. Cardiac tamponade is present in only $7 \%$ to $10 \%$ of traumatic intrapericardial diaphragmatic hernias. Associated injuries are frequent, and there is a predominance of rib fractures and head injury. ${ }^{3-5}$

Diaphragmatic hernia caused by blunt trauma is an uncommon cause of hemodynamic instability. Individuals with intrapericardial diaphragmatic hernia tend to show signs and symptoms of cardiac compression. Herniation of visceral contents into the pericardial sac reduces ventricular filling and stroke volume, and thus decreases cardiac output. Cardiac tamponade is an immediate sign in the large posttraumatic defect of the pericardium. ${ }^{1-6}$

The diagnosis of traumatic intrapericardial diaphragmatic hernia is considered on the basis of a chest $\mathrm{x}$-ray. Additional diagnostic tools are CT scan, contrast studies, and echocardiography. ${ }^{2,6,7}$ The surgical approach is abdominal in $80 \%$ of the intrapericardial diaphragmatic hernias diagnosed during the immediate posttraumatic period. The thoracic approach is used in $64 \%$ of the intrapericardial diaphragmatic hernias diagnosed in the delayed phase. The thoracic approach is preferred in patients with delayed traumatic 
intrapericardial diaphragmatic hernia, because adhesions in the pericardium can be freed more easily. ${ }^{3-6}$ In our case, an abdominal approach was used. The reduction of the hernia was performed without difficulties.

The organ most frequently involved in traumatic intrapericardial diaphragmatic hernia is the transverse colon, followed by the stomach and greater omentum. ${ }^{3}$ Most authors recommend the closing of the hernial defect with nonabsorbable sutures.

\section{CONCLUSIONS}

Traumatic intrapericardial diaphragmatic hernia alone can be fatal if it is not recognized and treated promptly. The observation of a diaphragmatic defect on cross-sectional imaging is essential to the diagnosis of traumatic intrapericardial diaphragmatic hernia. Once it is identified, immediate surgi- cal intervention is required to reduce the hernia and repair the defect.

\section{References}

1. Fleyfel M, Ferreira JF, Gonzalez De Linares H, et al. Cardiac tamponade after intrapericardial diaphragmatic hernia. Br J Anaesthesiol. 1994;73:249.

2. De Rooij PD, Haarman HJ. Herniation of the stomach into pericardial sac combined with cardiac luxation caused by blunt trauma: a case report. $J$ Trauma. 1993;34:453.

3. Reina A, Vidaña E, Soriano P, et al. Traumatic intrapericardial diaphragmatic hernia: case report and literature review. Injury. 2001;32:153-6.

4. Girzadas DV, Fligner DJ. Delayed traumatic intrapericardial diaphragmatic hernia associated with cardiac tamponade. Ann Emerg Med. 1991;20: 1246 .

5. Barker JA, Gavant ML, Hughes CB. Posttraumatic intrapericardial diaphragmatic hernia. Am J Roentgenol. 1997;169:315.

6. Kuzucu A, Isxik B, Baysal T, Soysal O, Ulutasx H. Traumatic intrapericardial diaphragmatic hernia. Radiography. 2007;13:169-71

7. Wenzel DJ, Hamilton JD. Cross-sectional CT of strangulating intrapericardial diaphragmatic hernia. AJR Am J Roentgenol. 2001;177:686-8.

\title{
Identification by transesophageal echocardiography of intramural hematoma and the site of aortic rupture
}

\author{
Kazumasa Orihashi, MD, Taijiro Sueda, MD, Kenji Okada, MD, and Katsuhiko Imai, MD, Hiroshima, Japan
}

Computed tomography (CT) is a noninvasive method of assessing the entire aorta and is essential for making a rapid diagnosis of acute aortic dissection with high sensitivity and specificity. ${ }^{1}$ On occasion, however, CT does not adequately inform a surgical decision, and additional information is needed. We report a case in which information obtained by transesophageal echocardiography (TEE) was critical in plotting a strategy.

\section{CLINICAL SUMMARY}

An 86-year-old female patient with sudden onset of flank pain was transferred to our hospital because of an extraperitoneal hematoma detected with regular CT. In the radiology department, her blood pressure dropped to $50 \mathrm{~mm} \mathrm{Hg}$. The CT examination, carried out with the blood pressure below $90 \mathrm{~mm} \mathrm{Hg}$, suggested left hemothorax; however, neither aortic dissection nor aneurysm could be recognized (Figure 1). The patient's blood pressure dropped to $40 \mathrm{~mm}$ $\mathrm{Hg}$, and she was immediately transferred to the operating

\footnotetext{
From the Department of Cardiovascular Surgery, Hiroshima University Hospital, Hiroshima, Japan.

Received for publication Nov 13, 2007; accepted for publication Nov 26, 2007.

Address for reprints: Kazumasa Orihashi, MD, Cardiovascular Surgery, Hiroshima University Hospital, Kasumi 1-2-3, Minami-ku, Hiroshima 734-8551, Japan (E-mail: orichan@hiroshima-u.ac.jp).

J Thorac Cardiovasc Surg 2008;136:1089-91

$0022-5223 / \$ 34.00$

Copyright (C) 2008 by The American Association for Thoracic Surgery doi:10.1016/j.jtcvs.2007.11.058
}

room with a diagnosis of hemothorax of unknown etiology and rupture site.

The TEE assessment revealed aortic dissection in the middle portion of the descending aorta, but with the proximal and distal segments intact (Figure 2, $A$ ). There was no aneurysm or thick atheroma. In the mediastinal hematoma, an echo-free area was present adjacent to the aorta (Figure 2, $B)$. The surrounding hematoma showed pulsatile motion. A color Doppler signal was not detected in either the false lumen or the echo-free area. TEE indicated localized type $\mathrm{B}$ dissection with mild thrombosis of the rupture site.

The left thorax was entered at the fifth intercostal space. Because the descending aorta was diffusely covered by hematoma, the extent of dissection could not be determined by inspection but instead was precisely located by TEEguided palpation. Under conditions of partial bypass and mild hypothermia, the aorta was crossclamped and incised. The false lumen was seen to be filled with fresh clot, and no intimal tear was found. The mediastinal hematoma at this level was not solid. A 5-cm segment of aorta was replaced with a vascular prosthesis. The patient's postoperative course was uneventful, without clamp injury or paraplegia, and she was discharged without complications.

\section{DISCUSSION}

Aortic dissection was not recognized with CT because the densities of aortic wall and blood were nearly identical. In 